\title{
Effect of induced blur on distance visual function
}

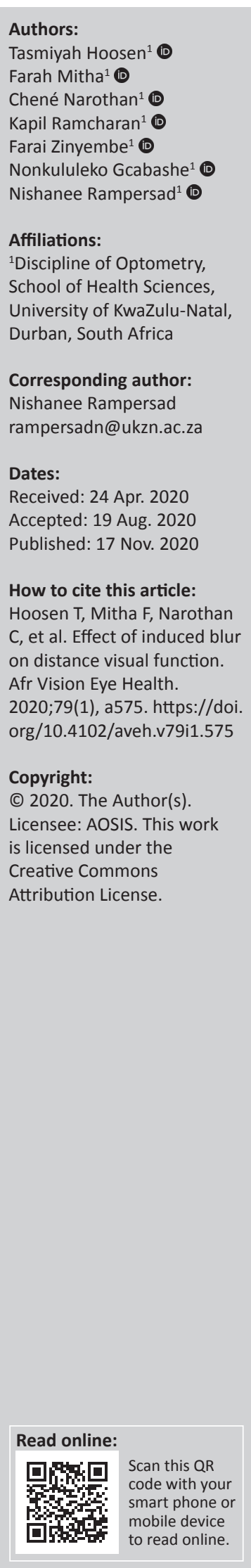

Background: Tests of visual function assess the eye and visual system. Factors such as ageing, refractive error, strabismus, amblyopia and ocular diseases have a negative effect on different visual functions.

Aim: To determine the effect of induced blur on three measures of distance visual function (visual acuity, stereopsis and contrast).

Setting: University of KwaZulu-Natal eye clinic.

Methods: A quasi-experimental research design was adopted and included 30 healthy young adults. Three convex test lenses (1 dioptre [D], $3 \mathrm{D}$ and 5 D) were used to induce blur and a lens of no power (0 D) was a placebo. The binocular visual acuity (BVA), stereoacuity and binocular contrast were assessed with the LogMAR chart, Howard-Dolman apparatus and Lea low contrast test, respectively. Tests of visual function were measured with no test lens (habitual measurement), and also with each test lens. Data were analysed using descriptive and inferential statistics.

Results: The mean habitual BVA and stereoacuity were $0.01 \log M A R \pm 0.05 \operatorname{LogMAR}$ and 29 arcsec \pm 20 arcsec, respectively. The mean BVA and stereoacuity measurements with the convex test lenses were $\geq 0.24 \operatorname{LogMAR}$ and $\geq 62$ arcsec, respectively and significantly different from the habitual measurements $(p<0.001)$. Most participants were unable to achieve the habitual contrast level with the three convex test lenses.

Conclusion: Induced blur reduced the three measures of distance visual function and the decrease was proportional to the power of the convex test lens. These findings should be considered when screening uncorrected ametropes for eligibility for a driver's licence.

Keywords: induced blur; visual acuity; stereoacuity; contrast; distance visual task.

\section{Introduction}

The term visual function describes how well the eye and visual system work and may be assessed with various tests in a clinical and/or research setting. ${ }^{1}$ Some of the tests of visual function include but are not limited to visual acuity, colour vision, stereoacuity, contrast sensitivity, perception of movement, dark adaptation and visual fields. ${ }^{2}$ The visual system is immature at birth and shows progressive improvement during the first few years of life. The age at which the different visual functions reach adult levels varies with some functions (motor fusion and stereopsis) showing adult levels after the first seven months of life whilst others (spatial acuity, contrast sensitivity and colour perception) continue to develop throughout the first few years of life and reach maturation around adolescence and early adulthood. ${ }^{3}$ Optimal levels of visual function are necessary for successful interaction with the visual environment to plan and execute vision-related tasks at distance and at near as well as to undertake activities of daily-living and independent mobility. ${ }^{4}$

Distance vision tasks refer to activities that are performed usually beyond 4 metres $(\mathrm{m}) .{ }^{4}$ Unlike near-vision tasks that are most often sedentary activities, distance vision tasks are related to mobility and include dynamic activities such as driving and engaging in sports as well as leisure activities. ${ }^{5}$ In a qualitative study that used methods such as focus groups, interviews, reflective journals and a questionnaire, to investigate the types of visual tasks performed at different distances, Atkinson et al. ${ }^{4}$ reported that driving was the most frequently mentioned distance vision activity. This is not surprising as driving is a favoured means of travel for many individuals worldwide. ${ }^{6}$ A systematic review reported that driving cessation is associated with poor quality of life, feelings of social isolation, reduced access to healthcare services as well as decreased physical and mental health. ${ }^{7}$ When interacting with the visual environment to undertake a 
distance vision task, different visual functions may be needed to safely and efficiently perform the task being undertaken. ${ }^{4}$

Some of the visual functions that are important for distance vision tasks include distance visual acuity (VA), stereoacuity and contrast sensitivity. Visual acuity, which is probably the most frequently measured aspect of visual function, is a measure of the spatial resolving ability of the eye. ${ }^{1,8}$ Visual acuity measurements may be assessed for different purposes and include determining the appropriate lens power in a refraction to help make decisions for prescribing and monitoring the progression of ocular conditions known to affect the optical and/or neural components of the visual system. ${ }^{9,10}$ Assessing VA is also important for determining eligibility for specific vision standards such as certification of driving licenses, visual disability pensions and visually impaired sporting codes. ${ }^{9,10}$ Stereopsis, which is considered as a hyperacuity, refers to the accurate binocular judgement of depth and distance and occurs because of retinal image disparity. ${ }^{11,12}$ The assessment of stereopsis is useful to screen for ocular conditions such as high uncorrected refractive error, anisometropia, aniseikonia, amblyopia and strabismus. ${ }^{13,14}$ Contrast sensitivity, which is the reciprocal of the minimum amount of contrast needed to see a target (contrast threshold), is a useful measure of visual function. ${ }^{15}$ Several physiological and pathological factors such as ageing, refractive error, strabismus, amblyopia and ocular diseases are known to influence the different aspects of visual function. ${ }^{11,15,16}$ Moreover, it is likely that these factors influence the different aspects of visual function differently ${ }^{10}$ and thus it is important to determine the effect of a specific factor whilst controlling for other factors. Accordingly, adopting such a methodology may help to better understand the extent of influence of a specific factor on the measures of visual function. Therefore, the aim of this study was to determine the effect of induced blur on some measures of distance visual function (binocular visual acuity [BVA], stereoacuity and contrast) in a sample with characteristics that helped to control for the effect of other physiological and pathological factors.

\section{Methodology}

The study used a quasi-experimental (one group pre-testpost-test) research design. The participants, consisted of undergraduate students attending the University of KwaZulu-Natal (UKZN), were recruited using convenience sampling and provided written informed consent prior to inclusion in the study. Tests used to assess eligibility for the study included case history, distance VA, refraction (objective and subjective), distance cover test and fusional vergence (if needed), ophthalmoscopy, slit lamp biomicroscopy and tonometry. Participants were aged between 17 and 25 years, had best corrected distance VA of at least 0.1 logarithm of the minimum angle of resolution (LogMAR) in each eye, spherical equivalent refractive error between 0.75 dioptre (D) and $-0.75 \mathrm{D}$ with no clinically significant anisometropia, distance orthophoria or compensated heterophoria and no history of any ocular, systemic and/or strabismus conditions.

Ophthalmic convex lenses were used to induce blur and this method of inducing optical defocus has been used in previous studies. ${ }^{13,16,17,18,19}$ The participant's vision was degraded using ophthalmic convex trial lenses that were placed in the trial frame that the participant wore during the data collection procedures as has been done previously. ${ }^{17}$ Four different test lenses $(0 \mathrm{D}, 1 \mathrm{D}, 3 \mathrm{D}$ and $5 \mathrm{D})$ were used in front of each eye in the trial frame to induce binocular blur. The $1 \mathrm{D}$ test lens was also used to induce monocular asymmetric blur. For standardisation, the $1 \mathrm{D}$ test lens was always placed in front of the participant's dominant eye for sighting, which was assessed using the 'hole-in-card' test ${ }^{20}$ when inducing the monocular asymmetric blur. The $0 \mathrm{D}$ test lens was used as a placebo in this study. Similar to the data collection procedure used in an earlier study, ${ }^{13}$ an artificial pupil was placed before each eye to control for image spread occurring as a result of the induced blur with the different test lenses. The artificial pupil, which was an opaque lens with a 4 millimetre $(\mathrm{mm})$ diameter, was further used to standardise the pupil size as it is well known that the latter influences the depth of focus, which can affect the different aspects of visual function. ${ }^{21}$

The tests used to collect data included distance VA, stereoacuity and contrast measured with a distance LogMAR chart, Howard-Dolman apparatus and Lea numbers low contrast $10 \mathrm{M}$ flipchart, respectively. The LogMAR chart is the gold standard for measuring VA in a research setting as it has a superior design based on scientific principles that address limitations identified in the Snellen VA chart. ${ }^{8,22}$ The range of distance VA measurements is between 1.0 LogMAR and $-0.3 \operatorname{LogMAR}$ when the chart is positioned $4 \mathrm{~m}$ away from the observer. ${ }^{22}$ Participants were instructed to verbalise the optotypes on the chart progressing in a downward direction and line scoring was used to determine the LogMAR VA measurement. ${ }^{22}$ The LogMAR chart with three different optotypes (letters, numbers and tumbling Es) were used in a random order to prevent memorisation of the optotypes during repeated testing.

The Howard-Dolman apparatus, which is an example of a real depth test, is the gold standard for measuring stereoacuity at intermediate and distant viewing distances as it allows for accurate stereoacuity measurements. ${ }^{14,23}$ Participants were instructed to adjust the position of the movable white vertical rod, using the strings in a pulley arrangement, such that it appeared the same distance away from the participant as the stationary white vertical rod. The participant's head was stabilised using a chin and forehead rest whilst fixating on the white vertical rods through the rectangular aperture in the Howard-Dolman apparatus to arrest any monocular cues to depth perception. ${ }^{23}$ Using the participant's interpupillary distance, a test distance of $3 \mathrm{~m}$ and the distance 
between the two white vertical rods, the stereoacuity was determined in arcsec using the formula:

$$
\begin{gathered}
\begin{array}{c}
\text { Interpupillary distance }(\mathrm{mm}) \times \text { distance between } \\
\text { two rods }(\mathrm{mm}) \times 206265
\end{array} \\
\hline \text { Test distance }{ }^{2}(\mathrm{~mm})
\end{gathered}
$$

which is provided in the Howard-Dolman apparatus. The stereoacuity measurements obtained with the HowardDolman apparatus were categorised as fine ( $\leq 60$ arcsec), moderate ( $>60$ arcsec but $\leq 200$ arcsec) or coarse/nil (> 200 arcsec) based on a classification system that has been used previously. ${ }^{24} \mathrm{~A}$ researcher reset the position of the movable white vertical rod after each stereoacuity measurement to minimise the influence of the previous measurement on the subsequent measurement.

The Lea numbers low contrast flipchart, which consists of five $10 \mathrm{M}$ number optotypes at five different contrast levels $(25 \%, 10 \%, 5 \%, 2.5 \%$ and $1.25 \%)$, was used to assess contrast. ${ }^{25}$ Participants were instructed to identify the number of optotypes being shown at the different contrast levels starting with the highest contrast level at a $3 \mathrm{~m}$ working distance. The Lea low contrast flipchart was held vertically and parallel to the participant's eyes to minimise the effect of tilting on the perception of the number of optotypes. ${ }^{25}$ The lowest contrast level at which the participant was able to correctly identify three or more of the optotypes was recorded as an indication of their contrast ability. Participants were instructed to read aloud the number optotypes either starting at the beginning or end of the line in a random order to minimise the effect of memorisation during repeated testing.

Three participants were examined simultaneously at the three data collection stations (distance acuity, stereopsis and contrast). Initially, the binocular distance VA, stereoacuity or contrast measurements were determined with no test lens and only with the participant's distance refractive correction (if needed) in the trial frame and this served as the habitual measurement. Thereafter, the binocular distance VA, stereoacuity or contrast measurements were repeated with each of the test lenses in the trial frame. The order in which the five test lenses were presented at each data collection station was randomised to minimise any bias. When six measurements (habitual and with the five test lenses) were completed at a data collection station, participants proceeded to the next station until all three participants had completed six measurements each at the three data collection stations. The order in which the three participants swopped amongst the three data collection stations was also randomised.

A pilot study was performed prior to data collection to refine and standardise the data collection procedure. Data collection took place in a room that consisted of fluorescent lighting and all windows were covered with venetian blinds to prevent any stray light from entering the room. Even though the room illumination was not measured in this study, none of the fluorescent bulbs were changed or replaced throughout the study and the setup of each data collection station remained unchanged. Two researchers were assigned to each data collection station and one researcher recorded the measurement whilst the other researcher presented the different test lenses in the trial frame. The researcher responsible for recording the measurements was unaware of which test lens was placed before the participants' eyes. In the same way, the researcher presenting the different test lenses was blinded to the measurements being recorded with the different test lenses. After all five measurements were taken with the test lenses, the two researchers combined their individual recordings to ensure that the different test lenses were matched with the different measurements taken at that data collection station. This was necessary as the two researchers at each data collection station were unaware of each other's recordings whilst the participant was being examined.

Data were captured and analysed using the Statistical Package for Social Sciences (SPSS version 25). The results are summarised using means, standard deviations, range, frequency counts and percentages. The paired sample $t$-test was used to compare the habitual and induced VA and stereoacuity measurements. A probability $(p)$ value of less than 0.05 was considered statistically significant.

\section{Ethical consideration}

Ethical clearance to conduct this study was received from the University of KwaZulu-Natal, School of Health Sciences Research Ethics Committee (SHSEC 028/15), 01 June 2015.

\section{Results}

The sample consisted of 30 participants comprising both females $(n=21)$ and males $(n=9)$. The mean age of participants was $20.77 \pm 1.50$ years with an age range of 18 to 23 years. The mean spherical equivalent refraction for the right and left eyes was $-0.08 \mathrm{D} \pm 0.35 \mathrm{D}$ (range, $+0.50 \mathrm{D}$ to $-0.75 \mathrm{D}$ ) and $-0.11 \mathrm{D} \pm 0.28 \mathrm{D}$ (range, $+0.50 \mathrm{D}$ to $-0.50 \mathrm{D}$ ), respectively. Just under half of the sample $(n=14)$ presented with orthophoria at distance whilst the remaining participants either had an exophoria $(n=12$, mean $=2.33$ prism dioptres $[\Delta] \pm 0.89 \Delta)$ or an esophoria $(n=4$, mean $=2.25 \Delta \pm 1.26 \Delta)$. The phorias were compensated based on the corresponding fusional vergence measurements. For the total sample, the mean habitual distance BVA was $0.01 \pm 0.05 \operatorname{LogMAR}$ with a range from $-0.10 \operatorname{LogMAR}$ to $0.10 \operatorname{LogMAR}$. The mean habitual distance BVA was similar in male (0.01 $\log M A R \pm 0.06 \log M A R)$ and female (0.01 $\log M A R \pm 0.05 \log M A R)$ participants. The mean habitual distance stereoacuity measurement for the total sample was 29 arcsec \pm 20 arcsec with a range from 4 to 81 arcsec. In contrast to the gender related trend observed for the habitual VA measurement, male participants had worse habitual stereoacuity than females (35 arcsec \pm 22 arcsec vs. $27 \operatorname{arcsec} \pm 20$ arcsec). All participants $(n=30)$ were able to correctly identify all five number optotypes at the lowest contrast level $(1.25 \%)$ when no test lens was placed in the trial frame. 
TABLE 1: Mean distance LogMAR BVA with the five test lenses used to induce optical defocus.

\begin{tabular}{lccc}
\hline Test lens & $\begin{array}{c}\text { Mean distance } \\
\text { BVA (LogMAR) }\end{array}$ & $\begin{array}{c}\text { Mean difference compared with } \\
\text { habitual distance LogMAR BVA (0.01) }\end{array}$ & $\boldsymbol{p}^{\dagger}$ \\
\hline OD & $0.01 \pm 0.06$ & 0.00 & 0.698 \\
1 D (asymmetric) & $0.03 \pm 0.07$ & 0.02 & 0.085 \\
1D & $0.24 \pm 0.16$ & 0.23 & $<0.001^{*}$ \\
3 D & $0.81 \pm 0.14$ & 0.80 & $<0.001^{*}$ \\
5D & $0.89 \pm 0.12$ & 0.88 & $<0.001^{*}$ \\
\hline
\end{tabular}

D, dioptre; BVA, binocular visual acuity.

*, $p$-value statistically significant.

$\dagger$, paired $t$-test.

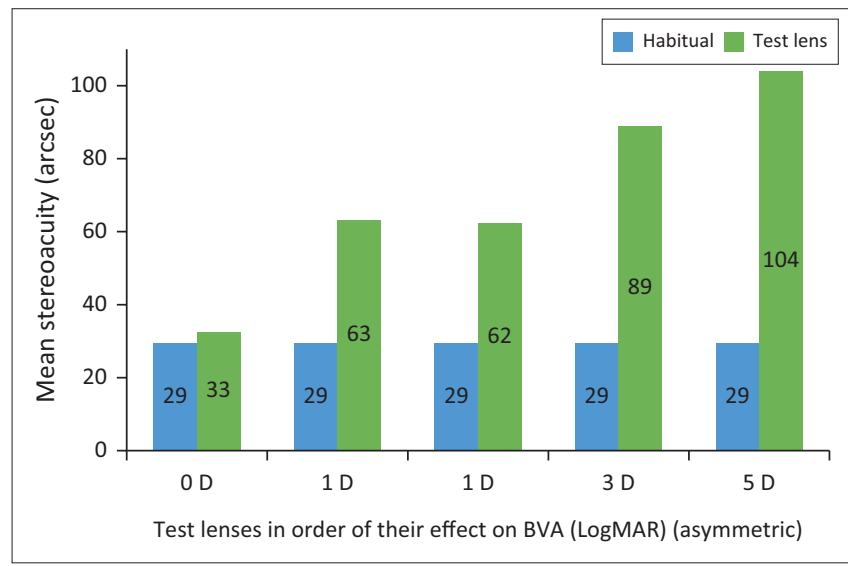

D, dioptre; BVA, binocular visual acuity.

FIGURE 1: Mean habitual distance stereoacuity and induced stereoacuity with the five test lenses used to induce optical defocus.

Table 1 shows the mean LogMAR BVA measurements obtained with the different test lenses used to induce the optical defocus and the mean difference in LogMAR BVA when compared with the habitual LogMAR BVA of 0.01 . As expected, the mean distance LogMAR BVA decreased as the power of the test lens that was used to induce the optical defocus increased (Table 1). The mean distance BVA measurement with the $0 \mathrm{D}$ and $1 \mathrm{D}$ (asymmetric) test lenses was similar to the habitual LogMAR BVA measurement with differences that were $\leq 0.02 \operatorname{LogMAR}(p \geq 0.08)$. In contrast, the other three test lenses resulted in mean LogMAR BVA measurements that were significantly different when compared with the habitual LogMAR BVA with the range of differences from 0.23 to 0.88 LogMAR $(p<0.001)$.

Figure 1 shows the mean habitual stereoacuity and the stereoacuity obtained with the different test lenses used to induce the optical defocus. The test lenses are arranged in order of their effect on the LogMAR BVA measurement, where the $0 \mathrm{D}$ and $5 \mathrm{D}$ test lenses had the least and greatest effects, respectively (Table 1). The mean stereoacuity with the $0 \mathrm{D}$ test lens was similar to the mean habitual stereoacuity with a difference of only $4 \operatorname{arcsec}(p=0.609)$. In contrast, the other four test lenses resulted in significantly worse mean stereoacuity measurements that were 33 arcsec to 75 arcsec higher than the mean habitual stereoacuity $(p<0.001)$. The $1 \mathrm{D}$ test lens in the monocular (asymmetric) and binocular conditions resulted in almost identical mean stereoacuity measurements (62 arcsec and 63 arcsec). Moreover, the mean stereoacuity measurement with the $1 \mathrm{D}$ test lens in these two

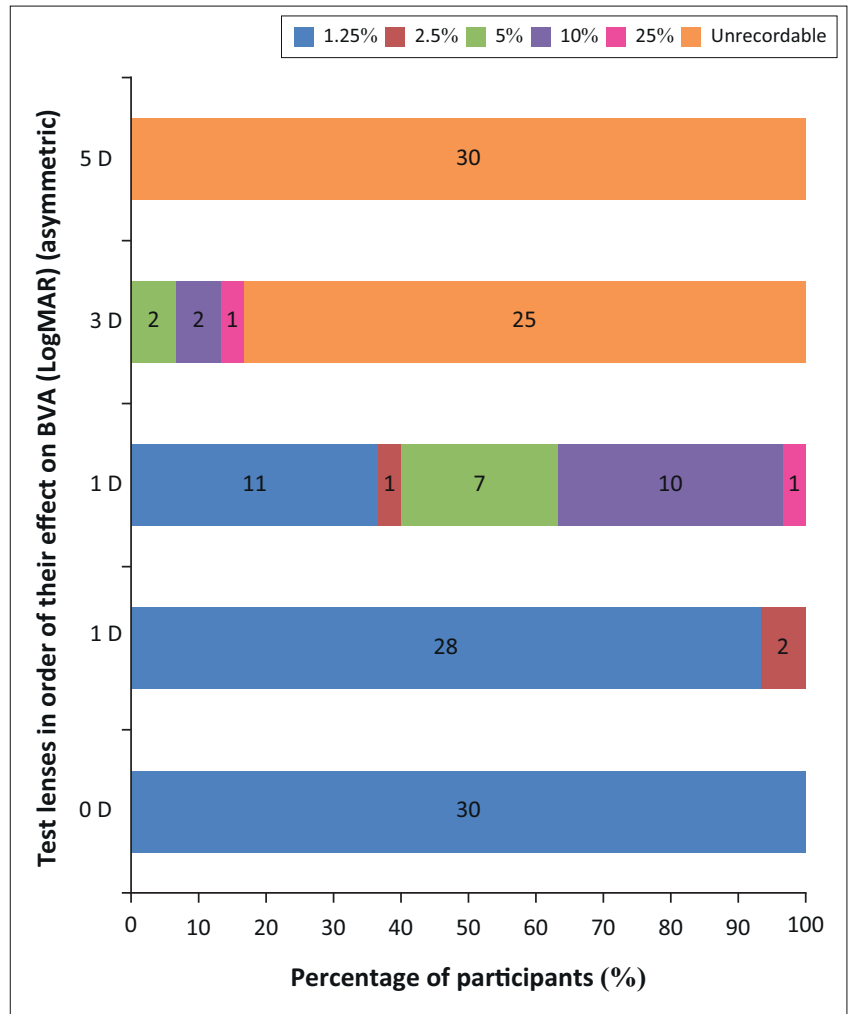

BVA, binocular visual acuity; D, dioptre.

FIGURE 2: Frequency of the different contrast levels with the five test lenses used to induce optical defocus.

conditions was more than two times the mean habitual stereoacuity (Figure 1). Similarly, the $3 \mathrm{D}$ and $5 \mathrm{D}$ test lenses resulted in mean stereoacuity measurements that were more than three times the mean habitual stereoacuity (Figure 1). In terms of the classification of stereoacuity measurements used in the present study, the habitual stereoacuity was categorised as fine stereoacuity and only the $0 \mathrm{D}$ test lens resulted in an induced stereoacuity measurement that could still be categorised as fine stereoacuity. In contrast, the mean stereoacuity measurements with the other four test lenses were categorised as moderate stereoacuity.

Figure 2 shows the frequency of the different contrast levels that were recorded with the five test lenses. All participants $(n=30)$ were able to correctly identify all five number optotypes at the $1.25 \%$ contrast level with the $0 \mathrm{D}$ test lens, which is identical to the results found when no test lens was placed in the trial frame. The majority of participants $(n=28)$ were still able to achieve the $1.25 \%$ contrast level when the $1 \mathrm{D}$ test lens was placed in front of only one eye (asymmetric). The $1 \mathrm{D}$ test lens placed in front of both eyes showed the most varied results as the majority of participants either achieved the $1.25 \%(n=11)$ or $10 \%(n=10)$ contrast levels whilst one participant each correctly identified the number optotypes at the $2.5 \%$ and $25 \%$ contrast levels. More than $80 \%$ of participants $(n=25)$ were unable to recognise any of the five number optotypes even at the highest contrast level (25\%) with the $3 \mathrm{D}$ test lens whilst the remaining five participants achieved some level of contrast that was $\geq 5 \%$ (Figure 2). None of the participants were able to achieve any of the 
contrast levels when the $5 \mathrm{D}$ test lens was placed in front of both eyes (Figure 2).

\section{Discussion}

This study aimed to determine the effect of induced blur on measures of distance visual function including BVA, stereopsis and contrast in a healthy young adult sample. Overall, the results showed that all three measures of distance visual function decreased as the power of the convex lens used to induce the blur increased. This finding was expected as defocus blur, which is because of inaccurate adjustment of the focal length, results in a loss of resolution of the details in an image. ${ }^{26}$ As the LogMAR VA chart assesses recognition acuity, ${ }^{10}$ the defocus blur induced, particularly with the high powered test lenses would have degraded participants' perception of the details (optotypes) in this test. This would also account for the trend observed when contrast was assessed with different convex test lenses as the details in this test are number optotypes. Even though the HowardDolman apparatus does not contain any optotypes, the decrease in stereoacuity is likely explained by both the loss of resolution and contrast as these two factors influence stereopsis. ${ }^{14,27}$ As much as a decrease in the measures of visual function was expected, using test lenses of different dioptric power would help to better understand what level of induced blur produces a substantial decrease in the distance acuity, stereopsis and contrast.

The mean LogMAR BVA measurement with the $1 \mathrm{D}$ (asymmetrical) test lens was similar to the mean habitual LogMAR BVA measurement with the mean difference equivalent to only one letter (0.02 LogMAR). This similarity in the mean BVA measurements may be as a result of the binocular VA measurement being more reflective of the VA in the non-dominant eye when the $1 \mathrm{D}$ test lens was placed before the dominant eye. This explanation is likely as the sample consisted of healthy young adults with no ocular anomalies and therefore the VA measurements in the two eyes are likely to be similar and correlated. ${ }^{28}$ The mean LogMAR BVA measurements when the $1 \mathrm{D}, 3 \mathrm{D}$ and $5 \mathrm{D}$ test lenses were placed binocularly before the participants' eyes were significantly different from the mean habitual measurement with the range from 0.23 to 0.88 LogMAR $(p<0.001)$. In addition, these differences are also clinically significant as they are higher than the maximum difference (0.2 LogMAR) that is suggested for inferring that an actual difference exists between two VA measurements when using a LogMAR VA chart. ${ }^{29}$ This implies that the two to almost nine-line difference in mean BVA measurements may be because of the effect of these test lenses and not the test-retest repeatability of the LogMAR VA chart. These results concur with the findings of other studies that also reported decreased LogMAR VA measurements when convex lenses were used to induce blur in healthy samples..$^{16,19,30}$

In the present study, the mean stereoacuity measurements with all test lenses except the $0 \mathrm{D}$ were significantly worse than the mean habitual stereoacuity. This finding is in agreement with previous studies that found reduced stereoacuity measurements when stereopsis was assessed using different methods (lenses or diffusing filters) to induce blur in participants with no ocular anomalies. ${ }^{16,17,30,31}$ As stereoacuity is a hyperacuity, it is sensitive to and negatively affected by defocus, reduced contrast and/or retinal image degradation. ${ }^{14}$ The $1 \mathrm{D}, 3 \mathrm{D}$ and $5 \mathrm{D}$ test lenses when placed binocularly before the participants' eyes, showed a linear decrease in stereoacuity as the power of the test lens increased. This finding is probably as a result of the corresponding linear increase in defocus and/or loss of contrast that occurred when the dioptric power of the convex test lenses increased. Moreover, these test lenses resulted in a substantial decrease in stereoacuity as the induced stereoacuity measurements, which were classified as moderate stereoacuity, were more than two times higher than the habitual stereoacuity.

Stereopsis is considered as the benchmark indicator of binocularity. ${ }^{11,27}$ Furthermore, unilateral blur causes a greater decrease in stereopsis than binocular blur. ${ }^{11,12,27}$ In cases of unilateral blur, factors such as anisometropia and its impact on fusion and stereopsis become important considerations in addition to defocus. ${ }^{17}$ Interestingly, the stereoacuity results were almost identical when the $1 \mathrm{D}$ test lens was used binocularly and before the dominant eye only. Thus, it can be speculated that $1 \mathrm{D}$ of monocular blur in the present study may not have had a major impact on fusion as the stereoacuity measurement was similar to the measurement obtained when the $1 \mathrm{D}$ test lens was worn binocularly. This implies that the reduced stereoacuity, noted when the $1 \mathrm{D}$ test lens was used to induce monocular blur, is most likely explained by the effect of defocus. An early study ${ }^{13}$ also found similar results as $0.5 \mathrm{D}$ to $1 \mathrm{D}$ of unilateral blur did not affect stereoacuity significantly when assessed with the Titmus and Randot stereo tests. It has been proposed that stereopsis is substantially affected with unilateral blur of $2 \mathrm{D}$ or more as this probably results in the blur-induced foveal suppression that impacts fusion and binocularity owing to the large interocular differences in VA. ${ }^{11,12,13,17,31}$ An alternate explanation for this finding could be related to the nature of the stereoscopic stimulus in the Howard-Dolman apparatus as studies have reported that low spatial frequency targets in real depth tests are less affected by unilateral blur. ${ }^{12,24}$

Contrast sensitivity, which assesses the ability of the visual system to detect images at varying luminance levels, can be measured with various clinical tests including the PelliRobson test, Lea low contrast test, Mars contrast sensitivity test and the Hiding Heidi test. ${ }^{15,25,31}$ As a result of limited availability of tests to assess contrast at the UKZN eye clinic, the Lea low contrast test was used to assess contrast in this study. Consequently, direct comparisons of the results from the present study to other studies are limited because studies have used different tests and presented results in different ways. Nevertheless, the trends seen in the results related to contrast were interesting and warrant discussion. For example, the small decrease in the frequency of participants that achieved the $1.25 \%$ contrast level with the $1 \mathrm{D}$ asymmetrical 
blur test lens, when compared with the habitual measurement, may be explained by disruption of binocular summation as a result of the induced unilateral blur. ${ }^{31,32}$ In addition, the majority of participants were unable to achieve any of the contrast levels with the $3 \mathrm{D}$ and $5 \mathrm{D}$ test lenses and this may be because the size of the $10 \mathrm{M}$ number optotypes at $3 \mathrm{~m}$ corresponds to a VA of 0.3 (which is $6 / 20$ or $0.52 \operatorname{LogMAR}) .^{25}$ As the mean VA measurements with the $3 \mathrm{D}$ and $5 \mathrm{D}$ test lenses were worse than $0.52 \operatorname{LogMAR}$, it implies that the size of the 10M optotypes were beyond the participants' spatial resolving ability with these test lenses and thus were unresolvable. Moreover, these high powered test lenses would have also degraded participants' perception of the $10 \mathrm{M}$ optotypes by increasing the width of the point spread function and consequently decreasing the contrast. ${ }^{32}$ All participants achieved some level of contrast when the $1 \mathrm{D}$ test lens was placed before both eyes and this may be accounted for by the mean VA with this test lens being $0.24 \operatorname{LogMAR}$, which is equivalent to approximately $6 / 10$ suggesting that the optotypes of the Lea low contrast test would have been resolved by participants. Despite this, the varied results with the $1 \mathrm{D}$ test lens before both eyes and the results of the other high powered lenses suggest that there is potential to use the Lea low contrast test as a simple screening test for detecting binocular refractive blur of $1 \mathrm{D}$ or more. This would be particularly useful when screening young children who resist monocular testing. As the Lea low contrast test is also available with Lea symbols, it would be suitable for children who are not familiar with or are unable to articulate letters or numbers.

The measures of distance visual function when assessed with the $0 \mathrm{D}$ test lens and the test lenses $(1 \mathrm{D}, 3 \mathrm{D}$ and $5 \mathrm{D})$ that were placed binocularly showed an interesting trend. With the $0 \mathrm{D}$ test lens, which served as the placebo, the mean LogMAR BVA was identical to the habitual LogMAR BVA and the mean stereoacuity was only 4 arcsec worse than the habitual stereoacuity and could still be categorised as fine stereoacuity. For the assessment of contrast, all participants correctly identified all five number optotypes at the lowest contrast level $(1.25 \%)$ with the $0 \mathrm{D}$ lens. Consequently, the $0 \mathrm{D}$ lens resulted in almost identical measurements for the three measures of visual function compared with the habitual. This finding suggests that the characteristics of the test lenses with the exception of dioptric power and corresponding change in centre thickness had a minimal effect on the measures of distance visual function. It was also noted that the measures of visual function decreased in proportion to the dioptric power of the convex test lenses where the $1 \mathrm{D}$ and $5 \mathrm{D}$ test lenses had the least and greatest effects, respectively. Other studies have also reported the same trend where measures of visual function were reduced with increasing lens power or density of diffusing filters. ${ }^{16,24,30}$ Even though the $1 \mathrm{D}$ test lens influenced acuity, stereopsis and contrast, the study findings suggest that the $3 \mathrm{D}$ and $5 \mathrm{D}$ test lenses resulted in a substantial decrease in all three measures of distance visual function. This is because the mean VA was eight lines worse whilst the mean stereoacuity was more than three times higher than the habitual measurements with these two test lenses. In addition, almost all participants were unable to achieve any level of contrast with the $3 \mathrm{D}$ and $5 \mathrm{D}$ test lenses. As a result, binocularinduced blur of $3 \mathrm{D}$ or more has the potential to substantially reduce distance visual function.

Driving is a complex visual task that involves various visual functions and processing for safe and efficient performance. ${ }^{6}$ To qualify for a motor cycle or light motor vehicle drivers licence (codes A1, A, B or EB) in South Africa, an individual requires a Snellen VA of at least 6/12 (equivalent to $0.3 \log$ MAR) and visual field of at least $70^{\circ}$ in each eye with or without refractive correction. ${ }^{33}$ If the individual is monocular or either the VA or visual field requirements are not meet in one eye, a Snellen VA of at least 6/9 (equivalent to $0.18 \log M A R$ ) and total horizontal visual field of at least $115^{\circ}$ is required in the other eye. ${ }^{33}$ To qualify for a heavy motor vehicle drivers licence (code C1, C, EC1 or EC) in South Africa, an individual requires a Snellen VA and visual field of at least 6/9 (equivalent to $0.18 \operatorname{LogMAR}$ ) and $70^{\circ}$ temporal, respectively, for each eye with or without refractive correction. ${ }^{33}$ In this way, the visual requirements for a driver's licence in South Africa are consistent with the trend in many other countries where VA and visual field are used to determine eligibility. ${ }^{6,34}$ The discussion related to visual field will be omitted as this aspect of visual function was not assessed in the present study. An early study found that peripheral visual fields were not affected by optical blur. ${ }^{18}$

The results of the present study in relation to the VA requirements for a South African drivers licence showed an interesting pattern. The $1 \mathrm{D}$ test lens when placed before both eyes resulted in a mean BVA of $0.24 \operatorname{LogMAR}$, which is equivalent to a Snellen VA that is between $6 / 10$ (0.2 LogMAR) and 6/12 (0.3 LogMAR). Consequently, this finding suggests that an individual with $1 \mathrm{D}$ of binocular blur is likely to meet the VA requirements for certification of a motor cycle or light motor vehicle drivers licence. However, the impact of $1 \mathrm{D}$ of binocular blur on the other two visual functions assessed in this study showed that stereopsis was more than two times worse than the habitual whilst more than $60 \%$ of participants were unable to achieve the same contrast level when no test lens was placed before the two eyes. As driving is a dynamic activity that is often undertaken in different luminance levels and at intermediate distances, optimal levels of contrast and distance stereopsis are important as they have a positive impact on driving performance. ${ }^{6,35}$ The results of this study imply that $1 \mathrm{D}$ of binocular blur may have a more substantial effect on distance stereopsis and contrast than VA if its impact is considered in relation to the demands of a task like driving especially at night or when it is raining and overcast. The problem of only assessing VA in a very specific environment (such as a clinical setting) and using this as a measure of an individual's ability to perform visual tasks like driving in a real-life setting has been described extensively. ${ }^{6,15}$ This is because a VA assessment with a high-contrast test chart may not always be reflective of an individual's ability to 
function in the real-life setting. 2,8,15 Thus, the results of this study support the claim that in addition to a high contrast VA measurement that other aspects of visual function should be incorporated into a more practical assessment of vision screening for the certification of a driver's licence. ${ }^{6,15,34}$ It is also recommended that dynamic VA, which is assessed in sports vision, be measured in future studies.

It is important to consider the methodology of the present study, particularly the characteristics of the sample and the method used to induce blur and the results related to the habitual visual function. Only healthy participants were included in this study to assess the effect of induced blur on measures of distance visual function as has been done in several other studies. ${ }^{10,16,24,31}$ The rationale for using a sample with specific characteristics was to enhance understanding of the effect of blur on acuity, stereopsis and contrast whilst controlling for other factors that are known to affect these measurements. ${ }^{16,24}$ This study used convex lenses to induce blur, which is consistent with the methods used in previous studies. ${ }^{10,13,16,17,18,19}$ Even though it is acknowledged that using convex lenses to induce blur may not share the same mechanism of blur that occurs because of ageing and/or pathological ocular conditions, the study findings provide insight into the effect on blur on acuity, stereopsis and contrast. Moreover, it may provide eye care personnel with a reasonable inference of what clinical measurements of stereopsis and contrast may be expected for a particular LogMAR BVA measurement. The mean habitual BVA and stereoacuity findings in this sample of young healthy adults is consistent with the findings of other studies involving normal samples. For instance, Subero et al. ${ }^{19}$ and Singh et al., ${ }^{30}$ who also studied the effect of induced blur using lenses on different visual functions, reported a mean best corrected VA of -0.01 and 0 LogMAR, respectively, which is similar to the VA in the present study $(0.01$ LogMAR). The mean stereoacuity in the present study was 29 arcsec and compares favourably with the mean of 20 arcsec reported by Odell et al. ${ }^{24}$ using the Frisby Davis distance real depth stereo test.

Strengths of the study include using a sample of healthy young adults and standardised data collection procedures that helped to control the influence of extraneous factors (such as monocular cues, age, ocular diseases, etc.) on the measures of acuity, stereopsis and contrast. Also, the study used randomised presentation of the test lenses, visual function tests and optotypes as well as reset the HowardDolman apparatus to minimise the impact of the learning effect when repeatedly assessing the different visual functions. This study has some limitations, which may influence the interpretations of the study findings. Firstly, the study only used spherical lenses and if cylindrical lenses were used to induce astigmatic or meridional blur it is likely that the effects on the measures of distance visual function would be different. Also the study was unable to separate the effect of defocus blur on VA and contrast as both these visual functions would have been simultaneously reduced when blur was induced. The study used convex lenses to induce blur and this may be different from habitual blur as a result of uncorrected ametropia. Therefore, it is recommended that future studies include individuals with habitual blur and emmetropes with induced blur so that a comparison can be made between the measurements of visual function in these two conditions of blur. Lastly, the testing procedures measured static visual function and were performed in a controlled clinical environment. Consequently, future studies should consider measuring these three visual functions dynamically and in a real-life environment.

\section{Conclusion}

The present study assessed the effect of induced blur on three measures of distance visual function including BVA, stereoacuity and contrast. Induced blur reduced all three measures of distance visual function and this decrease was proportional to the level of induced blur. As the low amounts of induced blur (1 D in the monocular and binocular conditions) also showed reduced distance visual function, it is recommended that small refractive errors be corrected as they have an impact especially on stereopsis and contrast. Both the Howard-Dolman apparatus and Lea low contrast test were sensitive to binocular and monocular blur and the findings related to these tests provide eye care personnel with a reasonable inference of the expected distance stereoacuity and contrast for a particular level of LogMAR BVA. This may be important especially when screening uncorrected ametropes for eligibility for a driver's licence as this usually involves only a high contrast VA measurement.

\section{Acknowledgements}

The authors acknowledge Mr. Nyika Mtemeri for assistance with the statistical analysis and Ms. Bongeka Vilakasi for assistance in the study.

\section{Competing interests}

The authors declare that they have no financial or personal relationships that may have inappropriately influenced them in writing this article.

\section{Authors' contributions}

T.H. was the study leader. N.G. and N.R. provided feedback on the study design. F.M., C.N., K.R. and F.Z. performed data collection. N.G. and N.R. wrote the manuscript.

\section{Funding information}

The research received no specific funding from any funding agency in the public, commercial or not-for-profit sectors.

\section{Data availability statement}

New and original data were collected, analysed and presented for this study. 


\section{Disclaimer}

The views and opinions expressed in this manuscript are those of the authors and do not necessarily reflect the official policy or position of any affiliated agency of the authors.

\section{References}

1. Colenbrander A. Visual functions and functional vision. Paper presented at: Vision 2005. Proceedings of the International Congress Series; Apr 4-7; London, United Kingdom, 2005; p. 482-486.

2. DeCarlo DK, Woo S, Woo GC. Patients with low vision. In: Benjamin WJ, editor Borish's clinical refraction. 2nd ed. Philadelphia, PA: Butterworth-Heinemann, 2006; p. 1591-1618

3. Siu C, Murphy KM. The development of human visual cortex and clinical implications. Eye Brain. 2018;10:25-36. https://doi.org/10.2147/EB.S130893

4. Atkinson MJ, Tally S, Heichel CW, Kozak I, Leich J, Levack A. A qualitative investigation of visual tasks with which to assess distance-specific visual
function. Qual Life Res. 2013;22(2):437-453. https://doi.org/10.1007/s11136function. Qua

5. Grosvenor T. Primary care optometry. 5th ed. St. Louis, MI: ButterworthHeinemann; 2007.

6. Owsley C, McGwin G. Vision and driving. Vision Res. 2010;50(23):2348-2361 https://doi.org/10.1016/j.visres.2010.05.021

7. Chihuri S, Mielenz TJ, DiMaggio CJ, et al. Driving cessation and health outcomes in older adults. J Am Geriatr Soc. 2016;64(2):332-341. https://doi.org/10.1111/jgs.13931

8. Elliot DB, Flanagan JG. Assessment of visual function. In: Elliot DB, editor. Clinical procedures in primary eye care. 4th ed. Philadelphia, PA: Elsevier, 2014; p. 32-67.

9. Bailey IL. Visual acuity. In: Benjamin WJ, editor. Borish's clinical refraction. 2nd ed. Philadelphia, PA: Butterworth-Heinmann, 2006; p. 217-246.

10. Leat SJ, Yakobchuk-Stanger C, Irving EL. Differential visual acuity - A new approach to measuring visual acuity. J Optom. 2020;13(1):41-49. https://doi.org/10.1016/j. optom.2019.04.002

11. Daum KM, McCormack GL. Fusion and binocularity. In: Benjamin WJ, editor Borish's clinical refraction. 2nd ed. Philadelphia, PA: Butterworth-Heinemann, 2006; p. 145-191.

12. Li RW, So K, Wu TH, et al. Monocular blur alters the tuning characteristics of stereopsis for spatial frequency and size. R Soc Open Sci. 2016;3(9):1-11. https:// doi.org/10.1098/rsos.160273

13. Lovasik JV, Szymkiw M. Effects of aniseikonia, anisometropia, accommodation retinal illuminance, and pupil size on stereopsis. Invest Ophthalmol Vis Sci. $1985 ; 26(5): 741-750$

14. Westheimer G. Clinical evaluation of stereopsis. Vision Res. 2013;90:38-42 https://doi.org/10.1016/j.visres.2012.10.005

15. Elliot DB. Contrast sensitivity and glare testing. In: Benjamin WJ, editor Borish's clinical refraction. 2nd ed. Philadelphia, PA: Butterworth-Heinmann, 2006; p. 247-288.

16. Costa MF, Moreira SMCF, Hamer RD, Ventura DF. Effects of age and optical blur on real depth stereoacuity. Ophthalmic Physiol Opt. 2010;30(5):660-666. https:// doi.org/10.1111/j.1475-1313.2010.00750.x
17. Dadeya S, Shibal F. The effect of anisometropia on binocular visual function. Indian J Ophthalmol. 2001;49(4):261-263.

18. Higgins KE, Wood J, Tait A. Vision and driving: Selective effect of optical blur on different driving tasks. Hum Factors. 1998;40(2):224-232. https://doi. org/10.1518/001872098779480415

19. Subero MS, Chamorro E, Cleva JM, Gonzalez A, Alonso J. Correlation between visual acuity and induced optical defocus: A new statistical approach. EC Ophthalmol. 2019;10(1):56-67.

20. Pointer JS. Sighting versus sensory ocular dominance. J Optom. 2012;5(2):52-55 https://doi.org/10.1016/j.optom.2012.03.001

21. Wang B, Ciuffreda KJ. Depth-of-focus of the human eye: Theory and clinical implications. Surv Ophthalmol. 2006;51(1):75-85. https://doi.org/10.1016/j. survophthal.2005.11.003

22. Hussain B, Saleh GM, Sivaprasad S, Hammond CJ. Changing from Snellen to LogMAR: Debate or delay? Clin Exp Ophthalmol. 2006;34(1):6-8. https://doi. org/10.1111/j.1442-9071.2006.01135.x

23. Cooper J. Stereopsis. In: Eskridge JB, Amos JF, Bartlett JD, editors. Clinical procedures in optometry. New York, NY: JB Lippincott Company, 1991; p. 121-134.

24. Odell NV, Hatt SR, Leske DA, Adams WE, Holmes JM. The effect of induced monocular blur on measures of stereoacuity. J AAPOS. 2009;13(2):136-141. https://doi.org/10.1016/j.jaapos.2008.09.005

25. Good-Lite Company. Lea contrast sensitivity [homepage on the Internet]. GoodLite Company; 1988. [cited 2015 Apr 23]. Available from: https://www.good-lite. com/cw3/Assets/documents/Lea\%20Contrast $\% 20$ Sensitivity-web.pdf

26. Tiwari S, Shukla VP, Biradar SR. Blur classification using wavelet transform and feed forward neural network. Int J M Educ Comp Sci. 2014;6(4):16-23. https://doi. org/10.5815/ijmecs.2014.04.03

27. Saladin JJ. Phorometry and stereopsis. In: Benjamin WJ, editor. Borish's clinical refraction. 2nd ed. Philadelphia, PA: Butterworth Heinemann, 2006; p. 899-962.

28. Armstrong RA. Statistical guidelines for the analysis of data obtained from one or both eyes. Ophthalmic Physiol Opt. 2013;33(1):7-14. https://doi.org/10.1111/ opo.12009

29. Bailey IL, Lovie-Kitchin JE. Visual acuity testing. From the laboratory to the clinic. Vision Res. 2013;90:2-9. https://doi.org/10.1016/j.visres.2013.05.004

30. Singh D, Aggarwal S, Sachdeva MM, Saxena R. Effect of induced monocular blur on monocular and binocular visual functions. Indian J Clin Exp Ophthalmol. 2015;1(4):197-201. https://doi.org/10.5958/2395-1451.2015.00015.3

31. Hairol MI, Arusulem L, Ying WJ. Effect of monocular blur on clinical measurements of stereopsis and binocular contrast sensitivity. J Sains Kesihatan Malaysia. 2017;15(1):19-25. https://doi.org/10.17576/JSKM-2017-1501-03

32. Pardhan S, Gilchrist J. The effect of monocular defocus on binocular contrast sensitivity. Ophthalmic Physiol Opt. 1990;10(1):33-36. https://doi.org/10.1111/ j.1475-1313.1990.tb01103.x

33. National Road Traffic Act 93 of 1996 [homepage on the Internet]. Republic of South Africa; 1996 [cited 2015 Oct 16]. Available from: https://www.gov.za/sites/ default/files/gcis_document/201409/act93of1996.pdf

34. Thorsland B, Strand N. Vision measurability and its impact on safe driving A literature review. Scand J Optom Vis Sci. 2016;9(1):1-9.

35. Bauer A, Dietz K, Kolling G, Hart W, Schiefer U. The relevance of stereopsis for motorists: A pilot study, Graefes Arch Clin Exp Ophthalmol. 2001;239(6):400-406. https://doi.org/10.1007/s004170100273 